\title{
Efecto bioinsecticida del extracto etanólico de las semillas de Annona cherimolia Miller "chirimoya" Y A. muricata Linneaus "guanábana" sobre larvas del IV estadio de Anopheles sp.
}

\author{
Larvicidal effectiveness of the ethanolic extract of Annona cherimolia \\ "cherimoya" Miller and A. muricata Linneaus "graviola" seeds against \\ Anopheles sp.
}

\author{
Bobadilla Álvarez, Miguel (1); Gina Zavaleta Espejo (1); Fanny Gil Franco (1); Luis \\ Pollack Velásquez (1); Manuel Sisniegas Gonzales (2)
}

\section{Resumen}

En vista del incremento de la resistencia a los insecticidas químicos frente al control de mosquitos vectores de enfermedades metaxénicas, es que se viene realizando la búsqueda de métodos alternativos, utilizando extractos de plantas con actividad larvicida debido a su capacidad de biodegradación generando menor daño ambiental. Bajo esta premisa, el objetivo del presente trabajo fue evaluar la mortalidad de larvas del IV estadio de Anopheles sp. mediante el extracto etanólico de las semillas de A. cherimolia (E1) y A. muricata (E2). Los mayores porcentajes de mortalidad, corregidos por la fórmula de Abbott, fueron de $100 \%$ a las 24 horas de exposición a la concentración de 0,8 y 0,12 ml/100 mL en E1 y E2, respectivamente, observándose un mayor efecto tóxico larvario a favor de E2 sobre E1 en $4,58 \%$ de mortalidad. El análisis probit mostró un patrón de respuesta heterogéneo de las larvas a las concentraciones letales al 50\% $\left(\mathrm{CL}_{50}\right)$ y al $90 \%\left(\mathrm{CL}_{90}\right)$ a lo largo de todos los tiempos de evaluación y una mayor homogeneidad a los tiempos letales al $50 \%\left(\mathrm{TL}_{50}\right)$ y al $90 \%\left(\mathrm{TL}_{90}\right)$ a medida que aumentaban las concentraciones de los extractos. Asimismo, la forma de las rectas de regresión muestran individuos larvarios con diferentes susceptibilidades a los extractos, lo que establece diferentes poblaciones o genotipos intervinientes. El trabajo permitió demostrar el efecto larvicida de ambas semillas y subraya la necesidad de realizar mayores ensayos in vitro como alternativa al control de insectos de importancia en salud pública.

Palabras clave: Bioinsecticida, semillas, mortalidad larvaria, Anopheles, Annona.

\begin{abstract}
In view of the increase of the resistance produced by chemical pesticides used against mosquitoes as vectors of public health diseases, searching for alternative methods has begun by using plant extracts with larvicidal activity which are environmentally safe and biologically degradable. Under this premise, the aim of the present study was to evaluate the mortality on fourth stage larvae of Anopheles $s p$. by using ethanolic extract of $A$. cherimolia (E1) and $A$. muricata (E2) seeds. The bigger mortality percentages corrected by Abbott's formula were $100 \%$ on the twelveth hour at 8,0 and $12,0 \mathrm{~mL} / 100 \mathrm{~mL}$ concentrations in E1 and E2. Morover E2 reached more larval toxic effect than E1 in about 4,58\% of mortality. The probit analysis showed an heterogeneous response of the larval individuals towards $50 \%\left(\mathrm{LC}_{50}\right)$ and $90 \%\left(\mathrm{LC}_{90}\right.$ ) lethal concentrations throughout the evaluation period, and an homogeneity response towards $50 \%\left(\mathrm{LT}_{50}\right)$ and $90 \%\left(\mathrm{LT}_{90}\right)$ lethal times when the concentrations of the extracts were increased. Likewise, the slopes of the log-dosage/probit lines showed larval individuals with different susceptibilities demonstrating the presence of different populations and gene compositions. The work allowed us to evaluate the efficiency of both extracts and to understand the necessity of more assays to ensure the best larvicidal control in such mosquitoes.
\end{abstract}

Key words: Biopesticide, seeds, larval mortality, Anopheles, Annona.

(1) Laboratorio de Zoología. Facultad de Ciencias Biológicas. Universidad Nacional de Trujillo.

Avenida Juan Pablo II s/n. Trujillo. Perú. e-mail: mcba@terramail.com.pe

(2) Departamento de Estadística. Facultad de Ciencias Físicas y Matemáticas. Universidad Nacional de Trujillo. 


\section{Introducción}

La malaria continúa siendo un importante problema de salud pública en numerosos países de la región centro y sur de América donde es considerada una enfermedad endémica de elevada prevalencia (Blanco et al., 2000), cuya distribución de casos en Latinoamérica hasta el año 1999, sitúa al Brasil como el país con el mayor número absoluto de casos de la enfermedad en un 50,5\% seguido por los países de la región subandina con 32,3\% (OPS, 2001). En la actualidad, las estrategias mundiales para prevenir y controlar la expansión de la enfermedad se fundamentan en la utilización de insecticidas químicos en el control del vector Anopheles, pero el inconveniente en su uso indiscriminado es el desarrollo de mecanismos de resistencia (INS/MINSA, 2000). En efecto, según la Organización Mundial de la Salud (1992) en América se ha demostrado la resistencia de especies vectores tales como $A$. albimanus, A. pseudopunctipennis, A. darlingi y $A$. vestitipennis hacia carbamatos, piretroides $\mathrm{y}$ organofosforados; este último grupo responsable de la resistencia en más de veinte especies de mosquitos a nivel mundial (Wirth, 2000) y es utilizado hoy en día en el Perú bajo el nombre comercial de abate (Temephos) en los programas de control larvario (Moquillaza, 1998), constituyendo un elemento de riesgo en salud pública debido a su toxicidad en humanos y persistencia en el ambiente (Gomero et al., 2000).

En muchos países, el empleo de controladores biológicos ha cobrado gran relevancia y se los considera con frecuencia alternativas ideales a los insecticidas (De Barjac, 1987). Se sabe por ejemplo de la capacidad infectiva del hongo Beauveria bassiana, del nematodo Romanomermis culicivorax (Frederickson, 1993), de la bacteria Bacillus thuringiensis var. Israelensis H-14 (Ventosilla et al., 2000) y de la capacidad predatora del crustáceo Chlamydoteca sp.(Torres et al., 2002), entre otros, sobre larvas de anofelinos. Asimismo, los productos naturales de origen vegetal están siendo investigados en cuanto a su actividad como repelentes en mosquitos adultos (Novak, 2000), y como intoxicantes e inhibidores del crecimiento frente a larvas, entre los que se encuentran el extracto de hojas de Ipomoea carneafistolasa eficaz en larvas y pupas de Anopheles gambiae (OPS, 1999), además de la actividad de Azadirachta indica (Alva y Boyer, 2000) y Lonchocarpus utilis (Mariños et al., 2000).

En este contexto, las especies del género Annona, además de controlar a insectos de importancia agrícola, también son efectivas sobre insectos de importancia médica tales como los mosquitos del género Anopheles (Rodríguez, 2000), Pediculus humanus, Pulex irritans, Cimex lectularius (Vásquez, 1944) y Blattella germanica (Alali et al., 1998), entre otros. Para esto se emplea diversos extractos orgánicos y no orgánicos de varias partes del vegetal (Jacobson, 1958), cuyos principios activos son considerados inhibidores del crecimiento (Gleye et al., 2000) y con efecto antialimentario comparables en actividad a los mostrados por la isoflavona rotenona (Guadano et al., 2000) e incluso superables en toxicidad a Azadirachta indica "nim" (Rodríguez, 2000).

Por esta razón, se hace necesario incentivar e incrementar la búsqueda de insecticidas naturales con especificidad en las especies de la familia Annonaceae y demostrar así su utilidad en salud pública para aportar mayores experiencias dirigidas al combate de insectos vectores de enfermedades al hombre, debido a su bajo costo, capacidad de biodegradación y como elemento racionalizador en el uso de insecticidas químicos, permitiendo de esta manera replantear nuevas estrategias a través del control selectivo de vectores propuesto por la Organización Mundial de la Salud (OMS, 2001). Bajo estas consideraciones, el objetivo del presente trabajo fue evaluar in vitro la mortalidad de larvas de Anopheles sp. empleando el extracto etanólico de las semillas de $A$. cherimolia Mill. "chirimoya" y $A$. muricata L. "guanabana", así como determinar la especie con mayor toxicidad larvaria. 


\section{Material y métodos}

\section{Obtención de larvas de Anopheles sp.}

Las larvas de Anopheles sp. se colectaron según la metodología propuesta por Ogosuko, (2002) a partir de criaderos naturales ubicados en las localidades de Santa Victoria y Menocucho, distrito de Laredo, provincia de Trujillo, departamento de La Libertad; luego se las trasladó al Laboratorio de Zoología de la Facultad de Ciencias Biológicas de la Universidad Nacional de Trujillo

\section{Crianza de larvas}

La crianza de larvas se realizó en fuentes de porcelana de $40 \times 28 \times 5 \mathrm{~cm}$ con agua potable declorinada reciclada interdiariamente. $\mathrm{La}$ alimentación a base de conejina esterilizada a $80^{\circ} \mathrm{C}$ se agregó una vez por día y era proporcional al número de larvas criadas (Ventosilla et al., 2002).

\section{Obtención de las semillas}

Las semillas de A. cherimolia y A. muricata se obtuvieron de un lote de frutos maduros seleccionados al azar en los mercados de la ciudad de Trujillo.

\section{Preparación de los extractos}

30 gramos del endospermo de las semillas fueron finamente cortados y macerados en 150 $\mathrm{mL}$ de etanol al $96 \%$ a temperatura ambiente durante una semana. Luego el solvente fue evaporado mediante baño maría a $50{ }^{\circ} \mathrm{C}$ y el extracto seco obtenido fue redisuelto en $30 \mathrm{~mL}$ de agua destilada a la misma temperatura de evaporación, obteniéndose una solución al $100 \%$ en peso del endospermo de las semillas por volumen de agua $(\% \mathrm{p} / \mathrm{v})$.

\section{Bioensayos}

Se utilizaron 825 larvas del IV estadio de Anopheles sp. cuya distribución fue en dos bloques: un bloque tratado con el extracto etanólico de las semillas de A. cherimolia (E1) y el otro bloque tratado con el extracto etanólico de las semillas de A. muricata (E2). Cada bloque se subdividió en 05 grupos expe- rimentales con un solo grupo testigo para ambos bloques, todos sometidos a tres repeticiones con 25 especímenes por repetición. A los grupos experimentales se les agregó 0,$01 ; 0,05$; 0,$1 ; 0,8$ y $1,2 \mathrm{~mL}$ de los extractos, respectivamente, aforados hasta $100 \mathrm{~mL}$ en agua destilada contenida en vasos de tecknopor de 250 $\mathrm{mL}$ de capacidad. Todos los grupos se mantuvieron con un rango de temperatura entre 23 y $26^{\circ} \mathrm{C}$ y humedad entre 75 y $80 \%$.

\section{Evaluación de la mortalidad}

Las lecturas de mortalidad se realizaron a la $1,6,12,24,36$ y 48 horas. Las larvas se consideraban muertas cuando no reaccionaban al momento de ser tocadas con un puntero romo en la región cervical (Consoli y Lourenço de Oliveira, 1994).

\section{Análisis estadístico}

Los cálculos de la mortalidad corregida se realizaron mediante la fórmula de Abbott en caso de muerte natural en el grupo testigo cuando éste era menor al $10 \%$. Las concentraciones letales al $50 \%\left(\mathrm{CL}_{50}\right)$ y $90 \%\left(\mathrm{CL}_{90}\right)$ y los tiempos letales al $50 \%\left(\mathrm{TL}_{50}\right)$ y $90 \%\left(\mathrm{TL}_{90}\right)$, así como los valores de las pendientes con sus limites de confianza, coeficientes de determinación y valores "chi" cuadrado $\left(\chi^{2}\right)$ se determinaron utilizando el EPA Probit Analysis Program Ver. 1.5.

El efecto unitario y de interacción de las variables se determinaron mediante un análisis de varianza bifactorial para un diseño de medidas de repetidas de observación y el mayor efecto tóxico larvario se calculó dividiendo la suma del total de larvas muertas en unidades probit de E2 sobre E1, expresados en porcentaje:

total de larvas muertas en unidades probit de

Eetal de larvas muertas en unidades probit de $\times 100$ E1

\section{Resultados y discusión}

La Tabla 1 muestra los porcentajes de mortalidad de las larvas del IV estadio de Anopheles sp. corregidas por la fórmula de Abbott. Dicha mortalidad fue total a partir de 
Tabla 1. Porcentaje de mortalidad corregida por la fórmula de Abbott de larvas del IV estadio de Anopheles sp. a diferentes concentraciones del extracto etanólico de las semillas de $A$. cherimolia y A. Muricata.

Extracto etanólico de las semillas de:

\section{A. cherimolia}

Concentraciones (mit
A. muricata

Concentraciones $(\mathrm{ml} / 100 \mathrm{~mL})$

Testigo

\begin{tabular}{|c|c|c|c|c|c|c|c|c|c|c|c|c|}
\hline \multirow{7}{*}{ 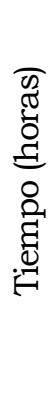 } & & 0,01 & 0,05 & 0,1 & 0,8 & 1,2 & 0,01 & 0,05 & 0,1 & 0,8 & 1,2 & \\
\hline & 1 & 0,0 & 1,0 & 3,0 & 4,0 & 5,0 & 0,0 & 3,0 & 5,0 & 8,0 & 11,0 & 0,0 \\
\hline & 6 & 1,0 & 15,0 & 19,0 & 59,0 & 81,0 & 16,0 & 19,0 & 35,0 & 65,0 & 68,0 & 0,0 \\
\hline & 12 & 23,0 & 45,0 & 57,0 & 82,0 & 99,0 & 25,0 & 31,0 & 61,0 & 96,0 & 99,0 & 0,0 \\
\hline & 24 & 49,0 & 75,0 & 82,0 & 100,0 & 100,0 & 61,0 & 73,0 & 84,0 & 100,0 & 100,0 & 0,0 \\
\hline & 36 & 53,0 & 82,0 & 88,0 & 100,0 & 100,0 & 64,0 & 79,0 & 96,0 & 100,0 & 100,0 & 4,0 \\
\hline & 48 & 57,0 & 96,0 & 97,0 & 100,0 & 100,0 & 85,0 & 97,0 & 99,0 & 100,0 & 100,0 & 4,0 \\
\hline
\end{tabular}

las 24 horas a las concentraciones de 0,8 y $1,2 \mathrm{~mL} / 100 \mathrm{~mL}$ en ambos extractos y superior al 50\% en las demás concentraciones a partir de las 36 horas de exposición. Además, el extracto etanólico de las semillas de $A$. muricata (E2) alcanzó mayor toxicidad en relación al extracto etanólico de las semillas de $A$. cherimolia (E1) en un 4,58\%, según el número total de larvas muertas en unidades probit, obtenido mediante la fórmula anteriormente indicada.

Los datos de concentraciones letales $\left(\mathrm{CL}_{50}\right.$ y $\left.\mathrm{CL}_{90}\right)$ y de tiempos letales $\left(\mathrm{TL}_{50}\right.$ y $\left.\mathrm{TL}_{90}\right)$ se encuentran en las Tablas 2 y 3 , respectivamente. A las seis horas de exposición, se empleó $0,41 \mathrm{~mL}$ de E1 y $0,314 \mathrm{~mL}$ de E2 para obtener una $\mathrm{CL}_{50}$ y 3,302 $\mathrm{mL}$ de E1 y 1,129 $\mathrm{mL}$ de E2 para obtener una $\mathrm{CL}_{90}$. En tanto a las 48 horas de exposición, se empleó 0,009 $\mathrm{mL}$ de E1 y $0,001 \mathrm{~mL}$ de $\mathrm{E} 2$ para conseguir una $\mathrm{CL}_{50}$ y se utilizó $0,038 \mathrm{~mL}$ de E1 y 0,016 mL de E2 para obtener una $\mathrm{CL}_{90}$. En cuanto a la concentración de $0,01 \mathrm{~mL} / 100 \mathrm{ml}$, se necesitó 31 h 28' empleando E1 y 19 h 43' haciendo uso de E2 para obtener un $\mathrm{TL}_{50}$, y $128 \mathrm{~h}$ 09' utilizando E1 y 74 h 47' recurriendo a E2 para conseguir un $\mathrm{TL}_{90}$. Del mismo modo con la concentración de 1,2 mL, se necesitó 3 h 13' empleando $\mathrm{E} 1 ; 3 \mathrm{~h} \mathrm{06}$ ' con E2 para obtener un $\mathrm{TL}_{50} ; 9 \mathrm{~h}$ 42' utilizando E1, y 7 h 26' con E2 para conseguir un $\mathrm{TL}_{90}$. Por lo tanto, se requerirá más cantidad de los extractos para producir mayor

Tabla 2. Valores de las concentraciones letales al $50 \%\left(\mathrm{CL}_{50}\right)$ y al $90 \%\left(\mathrm{CL}_{90}\right)$ del extracto etanólico de las semillas de $A$. cherimolia (E1) y A. muricata (E2) sobre larvas del IV estadio de Anopheles sp. a diferentes tiempos de evaluación.

\section{Tiempo (horas)}

\begin{tabular}{cccccc} 
& 6 & 12 & 24 & 36 & 48 \\
\cline { 2 - 6 } Extractos & \multicolumn{5}{c}{ CL (mL/100 mL) } \\
& CL50 - CL90 & CL50 - CL90 & CL50-CL90 & CL50 - CL90 & CL50 - CL90 \\
\hline E1 & $0,41-3,302$ & $0,070-1,021$ & $0,012-0,261$ & $0,008-0,118$ & $0,009-0,038$ \\
E2 & $0,314-12,9$ & $0,057-0,515$ & $0,004-0,367$ & $0,005-0,08$ & $0,001-0,016$ \\
\hline
\end{tabular}


Tabla 3. Valores de tiempos letales al $50 \%\left(\mathrm{TL}_{50}\right)$ y al $90 \%\left(\mathrm{TL}_{90}\right)$ del extracto etanólico de las semillas de A. cherimolia (E1) y A. muricata (E2) sobre larvas del IV estadio de Anopheles sp. a diferentes concentraciones.

\begin{tabular}{|c|c|c|c|c|c|c|c|c|c|}
\hline \multirow{3}{*}{ 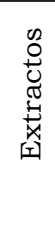 } & \multicolumn{9}{|c|}{ ConcentraciUn (mL/100 mL) } \\
\hline & \multicolumn{2}{|c|}{0,01} & \multicolumn{2}{|c|}{0,05} & \multicolumn{2}{|c|}{0,1} & \multicolumn{2}{|c|}{0,8} & 12,0 \\
\hline & TL50 & TL90 & TL50 & TL90 & TL50 & TL90 & TL50 & TL90 & TL50 TL90 \\
\hline E1 & $31 \mathrm{~h} 28 \mathrm{i}$ & 128 h 09i & 14 h 07î & 49 h 411 & 10 h 34î & 35 h $51 i$ & 5 h 021 & 16 h 49î & 3 h 13i 9 h $42 i$ \\
\hline E2 & 19 h 431 & 74 h $47 i$ & 13 h $28 \hat{1}$ & 42 h 40î & 7 h 501 & 29 h 30î & 3 h 381 & 10 h 50î & 3 h $06 i 17$ h $26 i$ \\
\hline
\end{tabular}

efecto tóxico larvario en menor tiempo y de menor cantidad del mismo para obtener una mayor toxicidad en mayor tiempo de exposición. Utilizando este criterio se consideró como de mayor toxicidad al extracto que necesite una menor concentración y tiempo para matar al $50 \%$ o $90 \%$ de la población expuesta (Silva y Casals, 2001), y en nuestro caso el extracto etanólico de las semillas de $A$. muricata fue el más indicado.

Como se observa en las tablas anteriores, a cada tiempo de evaluación correspondieron diferentes valores de $\mathrm{CL}_{50}$ y $\mathrm{CL}_{90}$, así como a cada concentración correspondieron también diferentes valores de $\mathrm{TL}_{50}$ y $\mathrm{TL}_{90}$ con sus respectivos datos sobre pendiente, desviación estándar, límites de confianza, $\chi^{2}$ tabulado y $\chi^{2}$ calculado, mostrados en la Tabla 4 y 5 , importantes para establecer respuestas y susceptibilidades de los individuos evaluados, los cuales fueron hallados mediante la transfor- mación Probit de aplicación muy extensa en toxicología y en ensayos biológicos (Mather, 1971). Este modelo implica llevar a logaritmo las distintas intensidades del estímulo y transformar la respuesta a unidades Probit (Silva y Casals, 2001). Los valores obtenidos para determinar si éstos se ajustan o no a dicha transformación se demostraron mediante el cálculo de los valores $\chi^{2}$ calculados los cuales fueron inferiores a los $\chi^{2}$ tabulados con un $\alpha=0,05$. En las mismas tablas se indican los valores de los intervalos de confianza y de las pendientes, cuyo recorrido no incluyó al valor cero, demostrando el efecto tóxico de los extractos sobre las larvas (Ventosilla et al., 2001). De otro lado, los valores propiamente dichos de las pendientes de la Tabla 4, indicaron susceptibilidad heterogénea de las larvas hacia las concentraciones letales al 50\% y 90\% a lo largo de todos los tiempos de evaluación. Caso contrario sucede con los valores de la pendien-

Tabla 4. Valores estadísticos de las concentraciones letales al $50 \%\left(\mathrm{CL}_{50}\right)$ y al $90 \%\left(\mathrm{CL}_{90}\right)$ del extracto etanólico de las semillas de A. cherimolia (E1) y A. muricata (E2) sobre larvas del IV estadio de Anopheles sp. a diferentes tiempos de evaluación.

\begin{tabular}{|c|c|c|c|c|c|c|c|c|c|c|}
\hline \multirow[b]{2}{*}{$\begin{array}{l}\text { Tiempo } \\
\text { (horas) }\end{array}$} & \multicolumn{2}{|c|}{$\mathrm{b}$} & \multicolumn{2}{|c|}{$\mathrm{S}$} & \multicolumn{2}{|c|}{ LC (95\%) } & \multicolumn{2}{|c|}{ E1 } & \multicolumn{2}{|c|}{ E2 } \\
\hline & E1 & E2 & E1 & E2 & E1 & E2 & $\begin{array}{l}\chi^{2} \\
\text { (c) }\end{array}$ & $\begin{array}{l}\chi^{2} \\
(\mathrm{t})\end{array}$ & $\begin{array}{l}\chi^{2} \\
\text { (c) }\end{array}$ & $\begin{array}{l}\chi^{2} \\
(\mathrm{t})\end{array}$ \\
\hline 6 & 1,4147 & 0,7943 & 0,1717 & 0,0957 & $1,0780-1,7514$ & $0,6066-0,9819$ & 5,194 & 7,815 & 3.819 & 7,815 \\
\hline 12 & 1,0990 & 1,3426 & 0,2103 & 0,2760 & $0,4301-1,7686$ & $0,4641-2,2211$ & 5,766 & 7,815 & 5.443 & 7,815 \\
\hline 24 & 0,9474 & 0,6511 & 0,2200 & 0,2135 & $0,5161-1,3787$ & $0,2325-1,0698$ & 0,059 & 3,841 & 0.620 & 3,841 \\
\hline 36 & 1,1058 & 1,0937 & 0,2275 & 0,4879 & $0,6599-1,5518$ & $0,1065-2,0809$ & 0,154 & 3,841 & 2.076 & 3,841 \\
\hline 48 & 2,0090 & 1,2158 & 0,3176 & 0,3638 & $1,3864-2,6315$ & $0,5026-1,9290$ & 1,132 & 3,841 & 0.027 & 3,841 \\
\hline
\end{tabular}

$\mathrm{b}=$ Valores de la pendiente $\mathrm{S}=$ Desviación estándar $\mathrm{LC}=$ Límites de confianza, $\chi^{2}(\mathrm{c})=$ "chi" cuadrado calculado, $\chi^{2}(\mathrm{t})=$ "chi" cuadrado tabulado. 
Tabla 5. Valores estadísticos de las tiempos letales al $50 \%\left(\mathrm{TL}_{50}\right)$ y al $90 \%\left(\mathrm{TL}_{90}\right)$ del extracto etanólico de las semillas de $A$. cherimolia (E1) y A. muricata (E2) sobre larvas del IV estadio de Anopheles sp. a diferentes concentraciones.

\begin{tabular}{ccc|cc|cc|cc|cc}
\hline $\begin{array}{c}\text { Conc. } \\
\text { (g/L.) }\end{array}$ & \multicolumn{2}{c}{$\mathrm{E} 1$} & \multicolumn{2}{c|}{$\mathrm{S} 2$} & \multicolumn{2}{c|}{ LC (95\%) } & \multicolumn{2}{c|}{$\mathrm{E} 1$} & \multicolumn{2}{c}{$\mathrm{E} 2$} \\
\hline 0,01 & 2,1019 & 2,2141 & 0,4348 & $\mathrm{E} 2,2331$ & $0,0937-4,1100$ & $1,7573-2,6710$ & 7,084 & 7,815 & 5,355 & 7,815 \\
0,05 & 2,5588 & 2,3702 & 0,2279 & 0,5210 & $2,1120-3,0057$ & $0,9238-3,8167$ & 8,192 & 9,488 & 7,442 & 9,488 \\
0,1 & 2,4179 & 2,2277 & 0,2115 & 0,1872 & $2,0032-2,8326$ & $1,8607-2,5947$ & 8,488 & 9,488 & 7,239 & 9,488 \\
0,8 & 2,4493 & 2,7023 & 0,2850 & 0,2755 & $1,8905-3,0081$ & $2,1621-3,2424$ & 0,104 & 3,841 & 3,689 & 3,841 \\
1,2 & 3,3695 & 2,6794 & 0,3271 & 0,6795 & $2,7284-4,0106$ & $0,9545-4,4042$ & 0,595 & 3,841 & 2,537 & 3,841 \\
\hline
\end{tabular}

$\mathrm{b}=$ Valores de la pendiente $\mathrm{S}=$ Desviación estándar $\mathrm{LC}=$ Límites de confianza, $\chi^{2}(\mathrm{c})=$ "chi" cuadrado calculado $\chi^{2}(\mathrm{t})=$ "chi" cuadrado tabulado.

tes de la Tabla 5, el cual muestra un mayor patrón de susceptibilidad y por lo tanto de homogeneidad de las larvas a los tiempos letales al $50 \%$ y $90 \%$ a medida que aumentaban las concentraciones de los extractos.

La forma de las rectas ajustadas, tal y como se observan en las Figuras 1a y b, indican la presencia de una sola población de individuos cuya sensibilidad se distribuye normalmente hasta las seis horas de exposición a los extractos, estableciendo homogeneidad en su composición genética (Montada et al., 1989). Sin embargo, después de las 12 horas de exposición (Fig. 1c) se observan ligeras inflexiones o partes horizontales que muestran los diferentes genotipos o poblaciones intervinientes con susceptibilidades disímiles a ambos extractos. La tendencia es repetitiva a medida del avance de los tiempos de evaluación, tal y como se aprecian en las Figuras 1d, e y f correspondientes a las 24, 36 y 48 horas, respectivamente, manteniéndose aún dichas inflexiones y partes horizontales. La situación anteriormente explicada, sumada a los valores bajos y un tanto diferentes de las pendientes enumeradas en la Tabla 6, establecen una respuesta biológica heterogénea de las larvas hacia las concentraciones de los extractos y estarían en relación directa con la procedencia geográfica de los individuos (Menocucho y Santa Victoria), los cuales fueron colectados a nivel de género, cuyos resultados podrían estar sobre o

Tabla 6. Valores numéricos de la pendiente (b) y del coeficiente de determinación $\left(r^{2}\right)$ de ambos extractos sobre larvas del IV estadio de Anopheles sp.

\begin{tabular}{|c|c|c|c|c|}
\hline \multirow[b]{2}{*}{$\begin{array}{l}\text { Tiempo } \\
\text { (horas) }\end{array}$} & \multicolumn{2}{|c|}{$\begin{array}{l}\text { Extracto etanólico de las } \\
\text { semillas de } A \text {. cherimolia }\end{array}$} & \multicolumn{2}{|c|}{$\begin{array}{l}\text { Extracto etanólico de las } \\
\text { semillas de } A \text {. muricata }\end{array}$} \\
\hline & $\begin{array}{l}\text { Pendiente } \\
\text { (b) }\end{array}$ & $\begin{array}{c}\text { Coeficiente de } \\
\text { determinación } \\
\left(\mathrm{r}^{2}\right)\end{array}$ & $\begin{array}{c}\text { Pendiente } \\
\text { (b) }\end{array}$ & $\begin{array}{l}\text { Coeficiente de } \\
\text { determinación } \\
\qquad\left(\mathrm{r}^{2}\right)\end{array}$ \\
\hline 1 & 0,7143 & $99,07 \%$ & 0,8202 & $96,65 \%$ \\
\hline 6 & 1,6254 & $98,70 \%$ & 0,8062 & $95,83 \%$ \\
\hline 12 & 1,3874 & $83,63 \%$ & 1,6913 & $93,15 \%$ \\
\hline 24 & 1,6375 & $96,74 \%$ & 1,5097 & $93,91 \%$ \\
\hline 36 & 1,5238 & $98,15 \%$ & 1,3998 & $94,70 \%$ \\
\hline 48 & 1,2262 & $\begin{array}{c}77,95 \% \\
\left(r^{2}\right)=92,37 \%\end{array}$ & 0,8640 & $\begin{array}{c}82,63 \% \\
\left(\mathrm{r}^{2}\right)=92,81 \%\end{array}$ \\
\hline
\end{tabular}



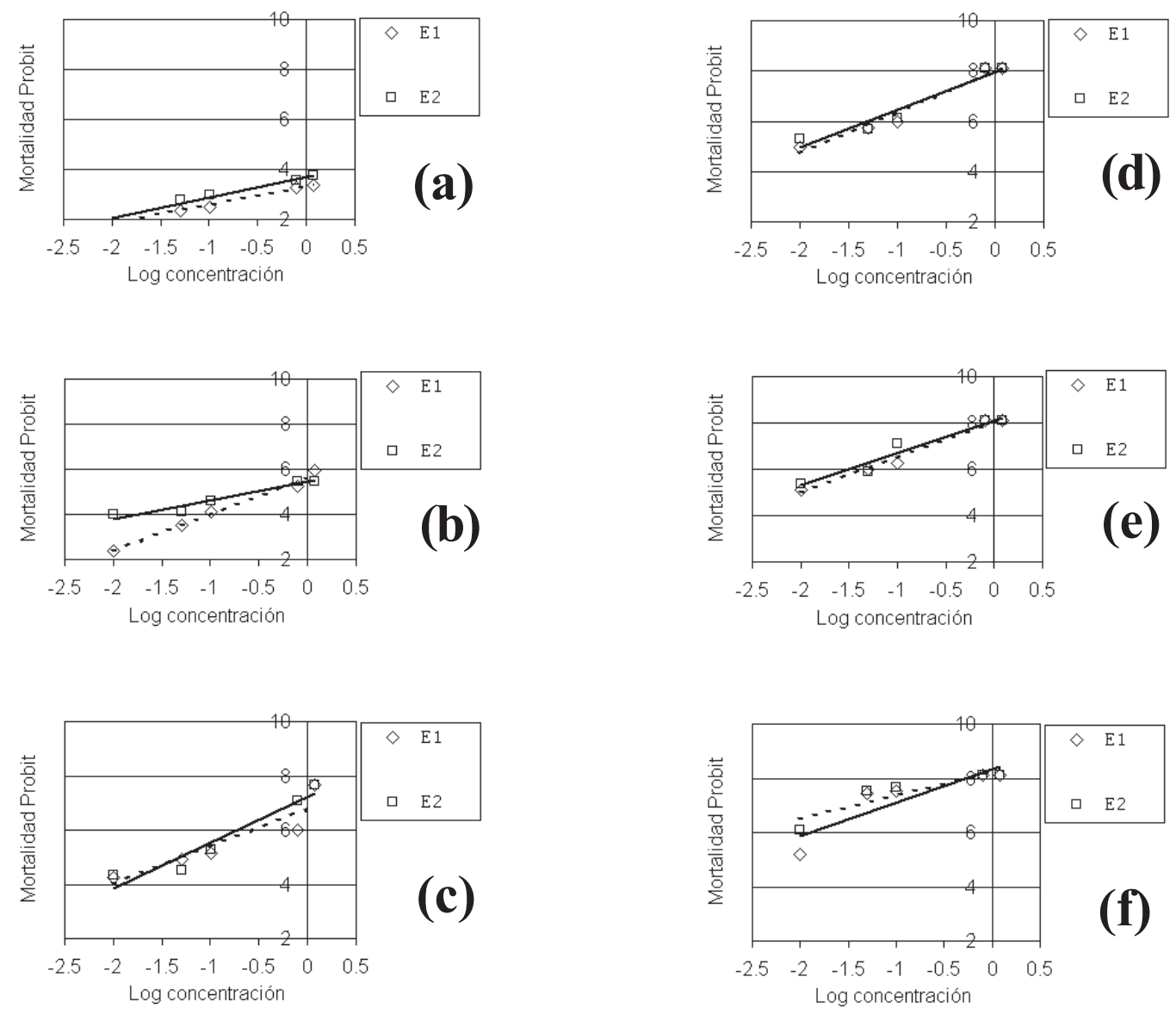

Figura 1. Recta de mortalidad probit de larvas del IV estadio de Anopheles sp. por el logaritmo de la concentración del extracto etanólico de las semillas de A. cherimolia (E1) y A. muricata(E2) a 1 hora de exposición (a), 6 horas de exposición (b), 12 horas de exposición (c), 24 horas de exposición (d), 36 horas de exposición (e) y a las 48 horas de exposición (f).

subdimensionando el estado real de la población evaluada (Silva y Casals, 2001). De otro lado, al observar los valores de los coeficientes de correlación $\left(\mathrm{r}^{2}\right)$ vemos una fuerte asociación entre ambas variables superior al 92\%; en consecuencia, la variabilidad de la mortalidad de las larvas puede explicarse por la exposición de éstas a las cinco concentraciones de los extractos desde los 60 minutos hasta las 48 horas, en tanto la diferencia ( $8 \%$ ) no podría explicarse por la misma regresión (Harnett y Murphy, 1987; Dawson-Saunders y Trapp, 1997).

El ensayo se vio afectado por factores tales como especie de semillas, concentraciones, tratamientos y unidades experimentales. Según esto, existen diferencias significativas entre las concentraciones y el efecto tóxico de las semillas, no siendo significativo el efecto de interacción entre estas dos variables. De igual manera, se establecieron diferencias altamente significativas entre los grupos experimentales (tratamientos) y los seis tiempos de evaluación, observándose que los grupos experimentales se comportan de manera diferente en cada tiempo, debido al incremento de la toxicidad o efecto acumulativo de los extractos conforme avanzaban los tiempos de evaluación. Dichos resultados son mostrados en la Tabla 7 mediante un análisis de varianza bifactorial para un diseño de medidas repetidas de observación. 
Tabla 7. Análisis de varianza bifactorial de un diseño en medidas repetidas de observación para los valores de mortalidad de larvas del IV estadio de Anopheles sp. utilizando el extracto etanólico de las semillas de $A$. cherimolia y $A$. muricata.

\begin{tabular}{|c|c|c|c|c|c|}
\hline $\begin{array}{l}\text { Fuente } \\
\text { de } \\
\text { variación }\end{array}$ & $\begin{array}{l}\text { Grados } \\
\text { de } \\
\text { libertad }\end{array}$ & $\begin{array}{l}\text { Suma de } \\
\text { cuadrados }\end{array}$ & $\begin{array}{c}\text { Cuadrados } \\
\text { medios }\end{array}$ & Razón F & $\begin{array}{c}\text { Valor de } \\
\text { probabilidad } \\
\mathrm{p}<0.05 \\
\text { dif. signif. } \\
\mathrm{p}>0.05 \\
\text { dif. no signif. }\end{array}$ \\
\hline Media & 1 & 5708,786 & 5708,786 & & \\
\hline Semillas & 1 & 2,856 & 2,856 & 4,7245 & 0,042 \\
\hline Concentraciones & 4 & 172,3273 & 43,081 & 71,257 & $1,5 \times 10^{-11}$ \\
\hline Semillas $\times$ Concentraciones & 4 & 1,769 & 0,442 & 0,731 & 0,582 \\
\hline Grupos & 9 & 176,952 & 19,661 & 32,519 & $9,4 \times 10^{-25}$ \\
\hline Tiempos & 5 & 437,178 & 87,435 & 384,754 & $1,4 \times 10^{-59}$ \\
\hline Grupos $\times$ Tiempos & 45 & 27,106 & 0,620 & 2,650 & $4,1 \times 10^{-05}$ \\
\hline Vasos dentro de grupos & 20 & 2,091 & 0,604 & 2,660 & $8,6 \times 10^{-04}$ \\
\hline Error experimental & 91 & 679 & 0,227 & & \\
\hline Total & 180 & 6382,795 & & & \\
\hline
\end{tabular}

En relación con lo anteriormente señalado, el efecto de los insecticidas vegetales es dependiente de algunos factores extrínsecos, tales como la especie y variedad de la planta, época de recolección, parte cosechada y forma de preparación, extracción y aplicación (Rodríguez, 1996). En nuestro caso, el material biológico empleado en los bioensayos fueron las semillas, reconocidas por utilizarse con mayor frecuencia en razón de su mayor toxicidad y por almacenar mayor cantidad de principios activos en relación con las otras partes del vegetal (Rodríguez, 2000). Asimismo, la extracción orgánica y redisolución del extracto seco en agua destilada fue para neutralizar los posibles efectos biológicos del solvente de extracción (Hoss, 1992). Ahora, entre los factores inherentes al organismo de prueba, es de destacar la variación de la susceptibilidad de acuerdo a la edad, estado de desarrollo, reorganización anatómica y a las variaciones propias de la muda; existe además, una tasa metabólica muy baja en individuos cercanos a la pupación (Lagunes y Villanueva citado por Silva y Casals, 1994), como las larvas.
De otro lado, la actividad tóxica de los principios activos de las annonas sería de ingesta y de contacto (Stoll, 1989; Rodríguez, 2000). De ingesta, porque al alimentarse las larvas mediante filtración y al no poseer una ingestión selectiva de partículas, los larvicidas pueden ingresar libremente produciendo toxicidad digestiva (Forattini citado por Consoli y Lourenço de Oliveira, 1994); y de contacto, mediante tres mecanismos interdependientes: transporte desde la cutícula al sitio de acción, inhibición enzimática y efecto sobre el sistema nervioso central, respiratorio u otro sistema involucrado como una consecuencia bioquímica del primer mecanismo (Gunther y Jeppson, 1962). En ese sentido, la actividad insecticida puede deberse a una interacción o efecto sinérgico de principios activos dada por un complejo alcaloidal benciltetrahidroisoquinoleínico (Chen et al.,2001) de acción citotóxica (Bruneton, 1994) y por acetogeninas, conocidas por tener propiedades insecticidas, antifúngicas y anticancerígenas (Alali et al., 1998); (Yao et al., 2000), cuya acción es la de inhibir a la NADH ubiquinona oxidoreductasa del complejo I mitocondrial de la cadena res- 
piratoria (Tormo et al., 1999), además de la acción de diterpenos del tipo kaurano conocidos por ser inhibidores del desarrollo de larvas (Valencia, 1994). En general, existen marcadas diferencias en la velocidad de acción de los principios activos en individuos de una misma especie de insectos, así como del grado de desarrollo de su sistema excretor y de la capacidad de almacenamiento de éstos (Gunther y Jeppson, 1962), dados por los túbulos de Malpighi y cuerpos grasos de los anofelinos, respectivamente (Consoli y Lourenço de Oliveira, 1994).

En general, los resultados sugieren que se deben realizarrealizar mayores estudios in vitro considerando ahora la diferenciación de especies para determinar susceptibilidades a cada una de ellas, empleando otros métodos de extracción, utilizando diferentes partes del vegetal o, en su defecto, realizar un screening o tamizaje fitoquímico empleando otras especies de la familia Annonaceae en especial con las autóctonas registradas en nuestra amazonía.

\section{Conclusiones}

- Se demostró el efecto bioinsecticida de ambos extractos sobre las larvas del IV estadio de Anopheles sp., siendo el extracto etanólico de las semillas de $A$. muricata más tóxico en relación al extracto etanólico de las semillas de $A$. cherimolia.

- El 100\% de la mortalidad de las larvas se efectuó a partir de las 24 horas a las concentraciones de 0,8 y $1,20 \mathrm{~mL} / 100 \mathrm{~mL}$ en ambos extractos.

- La respuesta de las larvas hacia los extractos demostró diferentes susceptibilidades y composición genética distintas.

\section{Literatura citada}

Alali, F.; Kaakeh, W.; Bennett, G. y McLaughlin, J. 1998. Annonaceous acetogenins as natural pesticides: Potent toxicity against insecticidesuceptible and-resistant German cockroaches (Dictyoptera: Blattellidae) J. Econ. Entomol. 91(3): 641-649

Alva, V. y A. Boyer. 2000. Prueba de susceptibilidad de larvas de mosquito Anopheles a Azadirachta. Libro de resúmenes de la XLII Convención Nacional de Entomología. Tarapoto. San Martín. Pp. 84-85.

Blanco, S.; A. Martínez; O. Cano; R. Tello e I. Mendoza. 2000. Introducción al Bacillus sphaericus cepa-2362 (GRISELEF) para el control biológico de vectores maláricos en Guatemala. Rev. Cub. Med. Trop. 52(1):37-43.

Bruneton, J. 1991 Elementos de fitoquímica y farmacognosia. Edit. Acribia. Zaragoza.

Consoli, R. y Lourenço de Oliveira, R. 1994. Principais mosquitos de importancia sanitária no Brasil. Edit. Fiocruz. Rio de Janeiro.

Dawson-Saunders, B. y R. Trapp. 1997. Bioestadística médica. 2. ${ }^{a}$ edición. Edit. El Manual Moderno S. A. de C. V. México D. F.

De Barjac, H. 1987. Operational bacterial insecticides and their potential for future improvement. En: Appl. Microbiol. Biotechnol. 21, 85-90.

Frederickson, E. 1993. Bionomia y control de Anopheles Albimanus. OPS/SMS. Cuaderno Técnico N. 34.

Gleye C.; Laurens A.; Hocquemiller R.; Laprevote O.; Serani L. and Cave A. 1997. Cohibins A and B, acetogenins from roots of Annona muricata. Phytochemistry. 44(8): 1541-1545

Gomero, L.; Y. Montoro y C. Caldas. 2000. Diagnóstico sobre el uso, manejo e impacto de los plaguicidas y otras sustancias tóxicas de uso doméstico en zonas urbano-marginales de Lima. Libro de Resúmenes de la XLII Convención Nacional de Entomología. Tarapoto. Perú.

Guadano, A.; Gutiérrez, C.; De La Peña, E.; Cortés, D. y González-Coloma, A. 2000. Insecticidal and mutagenic evaluation of two annonaceous acetogenins. J. Nat. Prod. 63(6): 773-776.

Gunther, A. y L. Jeppson. 1962. Insecticidas modernos y la producción mundial de alimentos. Compañía Editorial Continental S. A. México D. F.

Harnett, D. y J. Murphy. 1987. Introducción al análisis estadístico. Edit. Addison-Wesley Iberoamericana S. A. Delaware.

Hoss, R. 1992. Guía metodológica: Uso de extractos vegetales en la Regulación de Plagas. Cuaderno de Trabajo N. ${ }^{\circ} 01$. Red de Acción en Alternativas al Uso de Agroquímicos. Lima. Perú.

Jacobson, M. 1958. Insecticides from plants. A review of the literature (1941-1953). Agriculture Mined Book N. ${ }^{\circ}$ 154. US Government Printing Office. Washington.

Mariños, C.; J. Castro y D. Nongrados. 2000. Efecto biocida de Lonchocarpus utilis (Smith,1930) sobre Anopheles benarrochi 
(Gabaldón, 1941). Libro de Resúmenes del IV Congreso Peruano de Parasitología. Lima, pp. 246.

Mather, K. 1971. Análisis Estadístico en Biología. Edit. Paraninfo. Madrid.

Ministerio de Salud y Proyecto Vigía. 2000. Susceptibilidad de los Vectores de Malaria a los Insecticidas en el Perú. Ministerio de Salud. Lima.

Montada, D.; R. Tang; A. Navarro y F. García. 1989. Estudio de la Sensibilidad al Dimilín (Diflubenzurón) en una cepa de Aedes aegypti Linneaus, 1762 y de Culex quinquefasciatus Say, 1823 criadas en Laboratorio. Rev Cub. Med. Trop. 41(1): 56-63.

Moquillaza, J. 1998. Tratamiento focal con larvicidas en los criaderos de Aedes aegypti. En Entomología Básica: Uso de plaguicidas en salud pública y control vectorial integrado en malaria y OEM. Ministerio De Salud-Comunidad Europea. Yurimaguas. Perú.

Novak, R. 2000. The use of plant extracts as repellents for mosquitoes and biting flies. The abstract book of the $87^{\text {th }}$ Annual Meeting of the Amer. Mosq. Contr. Assoc. New Jersey, p. 44.

Ogosuko, E. 2002. Manual de complementación de vigilancia entomológica: División y vigilancia de artrópodos y roedores. Dirección General de Salud Ambiental. Lima.

Organización Mundial de la Salud. 1992. Resistencia de los Vectores de Enfermedades a los Plaguicidas. 15. ${ }^{\circ}$ Informe del Comité de Expertos de la OMS en Biología de Vectores y Lucha Antivectorial (Serie de Informes Técnicos N. ${ }^{\circ} 818$ ). Ginebra.

Organización Panamericana de la Salud. 1999. Control Selectivo de Vectores de Malaria. Guía para el nivel local de los sistemas de salud. Washington, DC. IV, 48 pp.

Organización Panamericana de la Salud. 2001. Situación de los programas de malaria en las Américas. Bol. Epid. Org. Panam. Salud. 22(1):10-
14.

Rodríguez, C. 1996. Extensión y capacitación en el uso de plaguicidas botánicos. En Memorias del I Taller Latinoamericano sobre Bioplaguicidas. El Zamorano. Honduras, pp.1-6.

Rodríguez, C. 2000. Plantas Contra Plagas: Potencial práctico de ajo, anona, nim, chile y tabaco. Edit. de la RAPAM. México. D. F.

Silva, G. y P. Casals. Bioensayo. Disponible en: http:// www.multired.com./ciencia/gosilagu/. Acceso: 14 de marzo de 2002.

Stoll, G. 1989. Protección natural de cultivos con recursos Provenientes de las granjas de las zonas tropicales y sub tropicales. Edit. Científica José Margraf. Alemania.

Tormo, J.; M. González; D. Cortés y E. Estornell. 1999. Kinetic characterization of mitochondrial complex I inhibitors using annonaceous acetogenins. Byochem. Byophys. 369(1):119-126

Torres, J.; F. García y A. Martin. 2002. Eficiencia de Chlamydoteca $s p$. como controlador biológico de larvas de Anopheles sp. a nivel de laboratorio. Libro de Resúmenes del XIV Congreso Nacional de Biología y VIII Simposio Nacional de Educación en Ciencias Biológicas. Tarapoto. Perú, pp 102.

Valencia, C. 195. Fundamentos de fitoquímica. Edit Trillas S.A. México D. F.

Vásquez, S. 1944. Estudio y comprobación insecticida de Annona squamosa peruana. Tesis para optar el título de químico farmacéutico. Universidad Nacional de Trujillo. Perú.

Ventosilla, P. y J. Chauca. 2000. Instructivo para el control de calidad de bioinsecticidas bacterianos. Instituto de Medicina Tropical "Alexander von Humboldt" Universidad Peruana Cayetano Heredia. Lima-Perú.

Ventosilla, P.; B. Infante; J. Merello y J. Chauca. 2001. Guía de Prácticas para la Producción de Bacillus thuringiensis subsp. israelensis usando alternativas locales para el control de vectores de enfermedades. OPS/OMS/ROW/ IMTAvH. Universidad Peruana Cayetano Heredia, p. 64.

Wirth, M. 2000. Temephos resistance in mosquito larvae: History \& consequence. The abstract book of the $87^{\text {th }}$ Annual Meeting of the Amer. Mosq. Contr. Assoc. New Jersey, p. 39. 